\title{
Comparison of NT-proBNP levels in hemodialysis versus peritoneal dialysis patients
}

\author{
Ondrej Ludka ${ }^{\mathrm{a}, \mathrm{c}}$, Jindrich Spinara, ${ }^{\mathrm{a}, \mathrm{c}}$, Josef Tomandl ${ }^{\mathrm{b}}$, Tomas Konecny ${ }^{\mathrm{c}, \mathrm{d}}$
}

\begin{abstract}
Background. Plasma N-terminal fragment of pro brain natriuretic peptide (NT-proBNP) concentration is elevated in cardiovascular diseases such as congestive heart failure, where increased levels of NT-proBNP indicate cardiac dysfunction, hypervolemia, and higher risk of hospitalization and death. These associations apply also to patients with severe impairment of kidney function. Little is known about diferences in plasma level of NT-proBNP in patients receiving hemodialysis (HD) versus those receiving continuous ambulatory peritoneal dialysis (CAPD).
\end{abstract}

Aim. To evaluate differences in plasma NT-proBNP concentration between HD and CAPD patients.

Methods. Plasma NT-proBNP concentration was prospectively measured in consecutive patients receiving either HD or CAPD at our hospital center. All other standard clinical parameters were recorded. The correlation between plasma NT-proBNP concentration and the type of dialysis was then examined.

Results. We studied 99 consecutive patients on HD (age $62 \pm 15$ years, $66 \%$ male) and 18 consecutive patients on CAPD (age $56 \pm 18$ years, $67 \%$ male). Both groups had similar baseline characteristics including duration of dialysis, left ventricular function and mass, and cardiothoracic ratio. Significantly more patients on HD had abnormal NTproBNP levels compared to patients on CAPD (97\% vs 44\%; $P<0.0001$ ), and this difference remained highly significant when using various NT-proBNP cut off values. A subgroup analysis revealed that the lower NT-proBNP levels of CAPD patients are most pronounced in patients with preserved left ventricular ejection function. As expected, NT-proBNP levels correlated negatively with left ventricular function and positively with cardiothoracic ratio, and this applied to both HD and CAPD groups.

Conclusion. The lower concentration of NT-proBNP in patients on CAPD compared to those on HD suggests that CAPD produces lesser hemodynamic stress, especially in patients with preserved left ventricular systolic function. Even though increased NT-proBNP levels have been shown to predict increased morbidity and mortality, further studies are necessary to assess the long term benefit of CAPD compared to HD.

Key words: hemodialysis, continuous ambulatory peritoneal dialysis, NT-proBNP, ejection fraction

Received: June 8, 2012; Accepted: October 23, 2012; Available online: November 13, 2012

http://dx.doi.org/10.5507/bp.2012.101

aInternal Cardiology Department, University Hospital Brno and Faculty of Medicine, Masaryk University, Brno, Czech Republic ${ }^{b}$ Department of Biochemistry, Faculty of Medicine, Masaryk University, Brno

IInternational Clinical Research Center, Brno

${ }^{d}$ Division of Cardiovascular Diseases, Mayo Clinic, Rochester, Minnesota, USA

Corresponding author: Ondrej Ludka, e-mail: oludka@fnbrno.cz

\section{INTRODUCTION}

A number of triggers, such as ventricular wall stretch, derangements in systemic blood pressure, increases in extracellular volume, or sodium shifts initiate the synthesis of pre-proBNP in left ventricular myocytes. Subsequently, the peptide is cleaved into proBNP which is secreted from myocytes and further cleaved by a membrane-bound serine protease into a biologically active B-type of natriuretic peptide (BNP) together with an inactive NT-proBNP. Compared with BNP, NT-proBNP has the advantage of having a longer plasma half-life (120 versus $20 \mathrm{~min}$ ) and lower biologic variation ${ }^{1-3}$. Numerous studies have demonstrated the potential value of NT-proBNP measurement in predicting abnormal left ventricular function in the general population ${ }^{46}$, in cardiac ischemia ${ }^{7-9}$, and in heart failure $^{10-13}$. In patients who were treated for acutely decompensated heart failure, BNP concentration decreased in parallel with fall in pulmonary capillary wedge pressure, suggesting that BNP may be able to mirror changes in fluid status and monitor therapeutic responses in heart failure $^{14}$. These findings are of particular relevance to patients who have end-stage renal disease on long-term HD or CAPD (ref..$^{15-19}$ ).

In our previous studies of HD patients we have shown that plasma levels of NT-proBNP do not vary according to the phase of the dialysis cycle as they likely reflect the long-term stress of the left ventricle. Similarly, NTproBNP concentrations do not seem to be affected by acute changes in volume or uraemic acid concentration during the dialysis cycle $^{20}$. Previous studies showed that successful kidney transplantation does normalise plasma concentrations of natriuretic peptides in the majority of the patients ${ }^{21,22}$.

Circulating NT-proBNP provides important information on cardiac dysfunction, hypervolemia, risk for hospi- 
talization, and even death, and this applies also to patients with severe impairment of kidney function. NT-proBNP is also a predictor of cardiovascular mortality in both HD and CAPD patients ${ }^{24}$. However, little is known about the diferences in plasma levels of NT-proBNP between patients receiving different types of dialysis. In our study, we aimed to investigate the differences in neurohumoral activation between end stage renal disease patients on HD and CAPD.

\section{MATERIAL AND METHODS}

\section{Patients}

Consecutive adult patients who have been on HD or CAPD for at least 3 months were recruited from a single regional dialysis center. Exclusion criteria included recent hospitalization (previous 30 days), clinical instability requiring urgent hospitalization, and the refusal to participate in the study. Patients on CAPD were recieving four exchanges per day using standard dialysis bags. The HD patients received regular dialysis using the high-flux membrane.

The clinical research ethics committee approved this study, and all subjects were consented and agreed to participate.

\section{Study design}

All of the patients underwent clinical evaluation (including vital sign, electrocardiography, chest X-ray and echocardiographic examination), and laboratory testing which included measurement of NT-proBNP levels. Hemodialysed patients underwent this evaluation on the day after regular haemodialysis, in other words, between two consecutive haemodialysis sessions.

\section{Biochemical analysis}

Blood sample was always collected after $20 \mathrm{~min}$ of supine rest. Basic laboratory tests were performed in a central hospital biochemical laboratory under standard conditions. Blood samples for NT-proBNP were centrifugated immediately after collection (15 min, 2700g) in $4{ }^{\circ} \mathrm{C}$, plasma was separated immediately thereafter and stored at $-70{ }^{\circ} \mathrm{C}$ until the time of analysis. NT-proBNP was assessed by means of heterogeneous immunoenzymatic method (ELISA-enzyme-linked immunosorbent assay), in our laboratory using commercially available immunoenzymatic sets by Biomedica $\mathrm{GmbH}$ with upper limit of normal values of $350 \mathrm{pmol} / \mathrm{L}$.

\section{Echocardiography}

Transthoracic echocardiography was performed using a Hewlett-Packard Sonos 5500, S3 probe (1.6- 3.2 MHz transducer). Basic echocardiographic parameters were obtained from M-mode, 2D and Doppler measurements in standard views (parasternal long axis, apical four and two chamber views). Left ventricle mass was calculated by the Penn convention. Ejection fraction was calculated using modified Simpson's rule.

\section{Statistical Analyses}

JMP version 8.0, Microsoft Excel and Statistica 6.0 for Windows were used for statistical analysis. All data are presented as the mean \pm standard deviation. Differences between groups were compared using Mann-Whitney's U-test when not normally distributed or unpaired Student's t-test when normally distributed. Categorical variables were compared using chi-square test. Relationships between NT-proBNP and variables were determined by linear regression analysis. A value of $P<0.05$ was considered statistically significant.

\section{RESULTS}

\section{Patient characteristics}

The study enrollment started in February 2001 and completed in May 2002. All 117 eligible patients were enrolled in this study, and their baseline demographic and clinical parameters are shown in Table 1 according to the type of dialysis they were receiving. There were no significant differences between the CAPD group and the HD group in the following categories: gender, age, duration of dialysis, heart rate, systolic and diastolic blood pressure, cardiothoracic ratio, and echocardiographic parameters including left ventricular mass, and left ventricular ejection fraction. We identified differences between the groups in the prevalence of hypertension (74\% versus $100 \%, P=0.0137)$, angiotensin-converting enzyme inhibitor use ( $51 \%$ versus $78 \%, P=0.0325)$, and lipid lowering medication use (26 versus $78 \%, P<0.0001$ ). The etiology of chronic kidney diseases was similar in HD patients versus CAPD patients (glomerulonephritis 27 versus $28 \%$, diabetic nephropathy 23 versus $22 \%$, tubulointersticial nephritis 18 versus $17 \%$, polycystic degeneration 9 versus $11 \%$, other 23 versus $22 \%$ ).

\section{NT-proBNP levels}

As seen in Panel A of Fig. 1, patients on HD were significantly more likely to have elevated levels of NTproBNP (>350 pmol/L) compared to patients on CAPD ( $97 \%$ vs. $44 \%, P<0.0001$ ). The number of patients with significantly elevated NT-proBNP (>1000 pmol/L) was also higher in the HD group ( $74 \%$ vs. $33 \%, P=0.0005$; Panel B of Fig. 1). When assessing the absolute values of NT-proBNP we note that the median was significantly higher in the HD group compared to the patients on CAPD (1870 pmol/L vs. 294 pmol/L), but the mean absolute values were similar in both groups (HD: $2281 \pm 1710$ pmol/L vs. CAPD: $1979 \pm 3107 \mathrm{pmol} / \mathrm{L} ; P=0.69)$.

\section{Difference in NT-proBNP and Ejection Fraction}

We performed an additional subgroup analysis of the levels of NT-proBNP after stratifying patients according to their left ventricular systolic function. As seen in Fig. 2 , when assessing patients with preserved left ventricular ejection fraction (LVEF) only, we saw a large difference in the number of patients with abnormal NT-proBNP between the HD and CAPD groups ( $96 \%$ vs $11 \%, P<0.0001)$. 


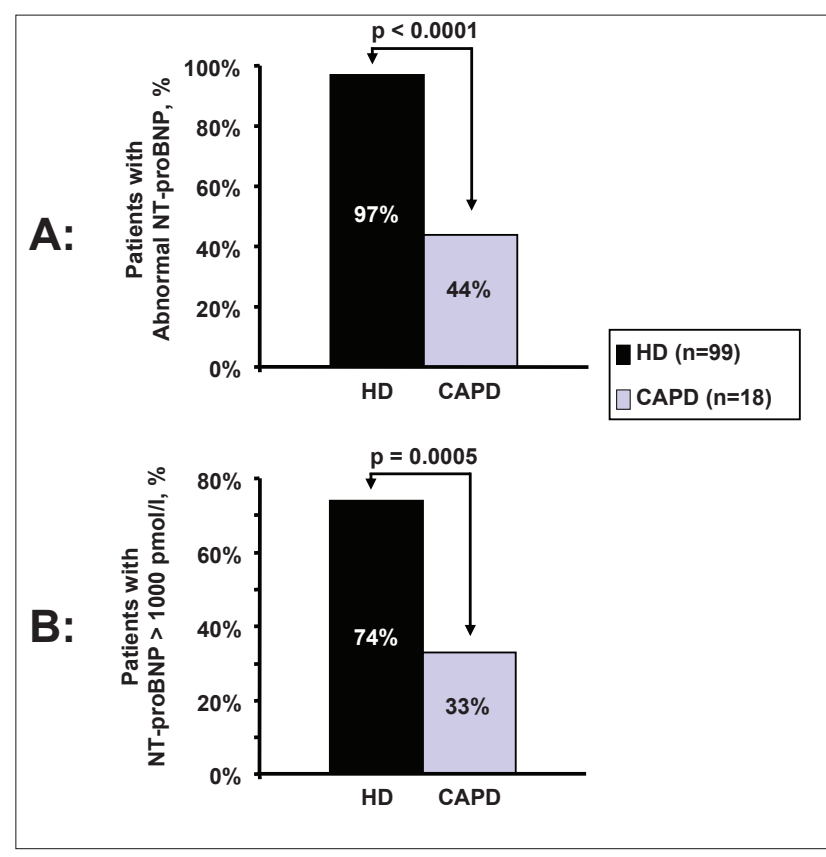

Fig. 1. Patients on hemodialysis (HD) are more likely to have elevated levels of NT-proBNP compared with patients on continuous ambulatory peritoneal dialysis (CAPD). This difference is highly significant at the recommended cut off value 350 $\mathrm{pmol} / \mathrm{L}$ (Panel A), and also when assessing higher cut off value $1000 \mathrm{pmol} / \mathrm{L}$ (Panel B).

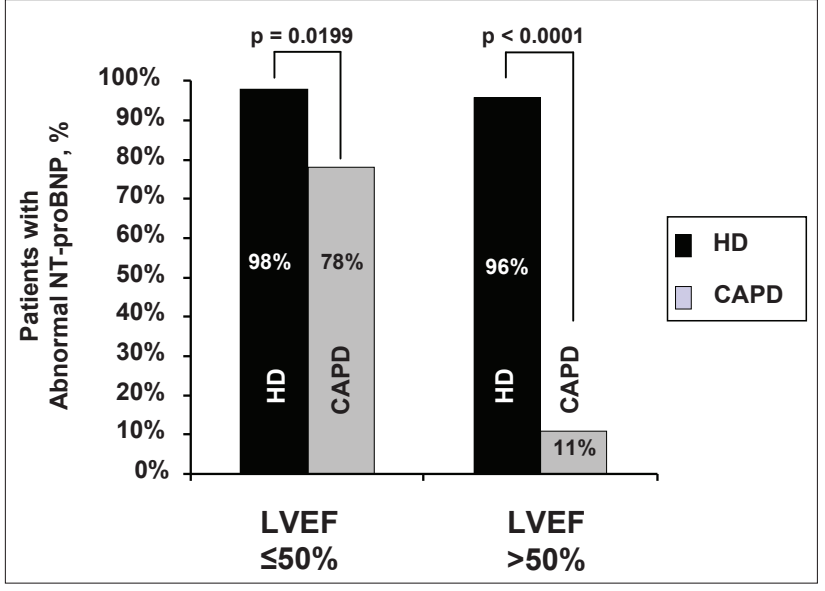

Fig. 2. Comparison NT-proBNP levels in patients on hemodialysis (HD) and continuous ambulatory peritoneal dialysis (CAPD). The two subgroups identified in this analysis are those with preserved left ventricular ejection fraction ( $\mathrm{LVEF}>50 \%$ ), and $\mathrm{LVEF} \leq 50 \%$.

Table 1. Baseline demographic and clinical parameters according to the type of dialysis.

\begin{tabular}{|c|c|c|c|}
\hline & HD patients $(n=99)$ & CAPD patients $(\mathrm{n}=18)$ & $P$ \\
\hline Male Gender (\%) & 66 & 67 & 0.9338 \\
\hline Age (year) & $62 \pm 15$ & $56 \pm 18$ & 0.1216 \\
\hline Duration of dialysis (month) & $41 \pm 51$ & $35 \pm 38$ & 0.5873 \\
\hline Hypertension (\%) & 74 & 100 & $0.0137 *$ \\
\hline Coronary artery disease (\%) & 42 & 44 & 0.8734 \\
\hline History of MI (\%) & 17 & 22 & 0.6075 \\
\hline History of stroke (\%) & 11 & 11 & 1.0000 \\
\hline Diabetes mellitus (\%) & 31 & 17 & 0.2081 \\
\hline ACEI/ARB (\%) & 51 & 78 & $0.0325 *$ \\
\hline $\mathrm{BB}(\%)$ & 44 & 67 & 0.0825 \\
\hline CCB (\%) & 41 & 61 & 0.1219 \\
\hline Diuretics (\%) & 60 & 83 & 0.0547 \\
\hline Insulin (\%) & 16 & 6 & 0.2402 \\
\hline Oral antidiabetics (\%) & 9 & 11 & 0.7871 \\
\hline Hypolipidemics (\%) & 26 & 78 & $<0.0001 *$ \\
\hline HR (per min) & $76 \pm 14$ & $72 \pm 15$ & 0.3219 \\
\hline $\mathrm{SBP}(\mathrm{mmHg})$ & $140 \pm 21$ & $143 \pm 12$ & 0.5113 \\
\hline DBP (mmHg) & $81 \pm 11$ & $82 \pm 10$ & 0.8836 \\
\hline $\mathrm{LA}(\mathrm{mm})$ & $41 \pm 6$ & $44 \pm 9$ & 0.2377 \\
\hline IVSd (mm) & $12 \pm 3$ & $11 \pm 3$ & 0.4175 \\
\hline PWd (mm) & $10 \pm 2$ & $10 \pm 2$ & 0.8819 \\
\hline LVDd (mm) & $58 \pm 9$ & $62 \pm 11$ & 0.2115 \\
\hline LVDs (mm) & $41 \pm 10$ & $43 \pm 12$ & 0.4708 \\
\hline LVEF (\%) & $53 \pm 10$ & $48 \pm 14$ & 0.1340 \\
\hline LVM (g) & $334 \pm 146$ & $377 \pm 250$ & 0.4861 \\
\hline CTR (\%) & $52 \pm 7$ & $51 \pm 8$ & 0.8165 \\
\hline
\end{tabular}


Table 2. NT-proBNP levels after stratifying patients according to their left ventricular systolic function.

\begin{tabular}{lccc}
\hline & HD - NT-proBNP $(\mathrm{pmol} / 1)$ & CAPD - NT-proBNP $(\mathrm{pmol} / \mathrm{l})$ & $P$ \\
\hline $\mathrm{EF} \leq 50 \%$ & $2886 \pm 1867$ & $3740 \pm 3666$ & 0.5134 \\
$\mathrm{EF}>50 \%$ & $1817 \pm 1417$ & $218 \pm 285$ & $<0.001$ \\
\hline
\end{tabular}

This difference was not as pronounced in patients with $\mathrm{LVEF} \leq 50 \%$ (98\% vs $78 \%, P=0.0199)$. Absolute values of NT-proBNP in this subgroup analysis also point at the largest difference being in patients with preserved LVEF (Table 2). Among patients with preserved LVEF, those on HD and CAPD had statistically similarly sized left atria (39 mm vs. $39 \mathrm{~mm}, P=0.9244$ ), similar burden of diastolic dysfunction ( $62.5 \%$ vs. $66.7 \%, P=0.8089)$, and similar left ventricular mass ( $293 \mathrm{~g}$ vs. $232 \mathrm{~g}, P=0.1384$ ). As expected, plasma level of NT-proBNP positively correlated with the cardiothoracic ratio (for patients on HD: $\mathrm{r}_{\mathrm{s}}=0.376$, $P<0.001$; for patients on CAPD: $\mathrm{r}_{\mathrm{s}}=0.713, P=0.001$ ), and negatively correlated with ejection fraction (for patients on HD: $\mathrm{r}_{\mathrm{s}}=-0.391, P<0.001$; for patients on CAPD: $\mathrm{r}_{\mathrm{s}}=$ $-0.708, P=0.001)$ in both HD and CAPD patients.

\section{DISCUSSION}

The main results of the study are: 1) patients on HD are significantly more likely to have elevated levels of NT-proBNP compared to patients on CAPD; 2) it is particularly patients with preserved left ventricular function in whom the difference in NT-proBNP between HD and CAPD is most pronounced. Since elevated NT-proBNP functions as a marker of adverse prognosis, these findings may have important clinic implication when considering the most appropriate type of dialysis especially in patients with normal left ventricular function.

\section{CAPD as an alternative to $\mathrm{HD}$}

Continuous ambulatory peritoneal dialysis is an important method for the treatment of patients with endstage renal disease comprises of diffusion and filtration in which peritoneum functions as a dialytic membrane. Dialytic solution is infused into the peritoneal cavity through a peritoneal catheter and is left inside for several hours according to the type of dialysis. Peritoneal dialysis has distinct indications and contraindications. Among common indications are difficult placement of an A-V shunt, type 1 diabetes mellitus, childhood and elderly patients. Contraindications include adhesions in the peritoneal cavity, active bowel disease, large hernia, colostomy, clinically significant ascites. Patients on CAPD enjoy increased freedom of movement, and minimal blood loss; disadvantages include increased bathing restrictions, the presence of a permanent catheter, higher rate of infectious complications, and a considerable amount of fluid in the peritoneal cavity ${ }^{23}$.

\section{NT-proBNP}

Natriuretic peptides (BNP and NT-proBNP) function as a markers of volume status and cardiovascular risk in end-stage renal disease patients ${ }^{24}$. Circulating NT-proBNP provides clinically important information on cardiac dysfunction, hypervolemia, and risk for hospitalization or death in patients with severe impairment of kidney function, and this is true for both HD and CAPD patients ${ }^{24,25}$. Both BNP and NT-proBNP are eliminated during hemodialysis, but their clearance varies depending on the dialysis membrane. BNP is cleared by both high- and low-flux membranes, with high-flux membranes giving higher clearance (due to mass balance) and reduction rates. NTproBNP has clearance and reduction rates similar to BNP when high-flux membranes are used but very low clearance with low-flux membranes, leading to an increase in postdialysis plasma concentrations. This may be explained in part by the different molecular masses of BNP (3.5 $\mathrm{kDa})$ and NT-proBNP $(8.5 \mathrm{kDa})\left(\right.$ ref. $\left.^{26}\right)$. The inter-dialysis variability of NT-proBNP was previously studied in our dialysis centre in 22 patients with the conclusion that the plasma level of NT-proBNP were practically unchanged on the various days of the dialysis schedule ${ }^{20}$.

In our study NT-proBNP was assessed by using commercially available sets with upper limit of normal values of $350 \mathrm{pmol} / \mathrm{L}$, but to confirm the validity of our results we tested our hypothesis also using a higher cut off value of $1000 \mathrm{pmol} / \mathrm{L}$, and the results remained unchanged.

\section{Abnormal NT-proBNP in HD vs. CAPD}

The effect of HD and CAPD on the cardiovascular system are significantly different, as HD is associated with major volume shifts while CAPD is not. The thought that such volume shifts would have adverse effect on the cardiovascular system have been postulated before, and there is an accumulating body of evidence suggesting that BNP and NT-proBNP levels are higher among patients on HD (ref. ${ }^{27-30}$ ). No previous studies to our knowledge assessed NT-proBNP according to the level of preservation of the left ventricular systolic function. Our results clearly show that patients on HD are more likely to have elevated levels of NT-proBNP compared to patients on CAPD, and this finding may suggest that from the cardiovascular stand point CAPD is a better alternative to HD for renal replacement therapy. These data are consistent with previously mentioned studies ${ }^{27-29}$. Whether an advantage predicted by a marker such as NT-proBNP leads to an actual morbidity or mortality benefit cannot be determined from the present study, and this clinically relevant hypothesis warrants further investigation. 


\section{Patients with preserved LVEF}

The novel finding of this study includes the comparison of plasma levels of NT-proBNP between subgroups according to the LVEF. This subgroup analysis showed that the most significant difference in plasma levels of NT-proBNP existed in patients with preserved ejection fraction (above 50\%), while only a modest difference was observed in patients with $\mathrm{LVEF} \leq 50 \%$. Similar finding was observed in patients with cardiothoracic ratio $\leq 50 \%$. The lower NT-proBNP concentration in CAPD patients as compared with HD patients suggests that cardiac load in CAPD patients may be lower than that in HD patients. Given that this difference is more pronounced in those with preserved LVEF we could hypothesize that these patients with LVEF > 50\% would be the once who could benefit from using CAPD most profoundly. The design of our exploratory study, however, allows only for hypothesis forming observation in this regard. To apply our findings into clinical practice we postulate that the lack of repetitive fluid shifts and sudden changes in cardiac hemodynamics could be taken into account when selecting the most appropriate dialysis modality, particularly in patients with preserved LVEF. Randomized studies would be needed to prove whether the use of CAPD compared to HD could have a beneficial effect on cardiovascular and mortality end points, as well as possibly lead to fewer hospitalization and improved quality of life.

\section{LIMITATIONS}

The design of a cross-sectional analysis carries many well known inherent limitations. The selection for HD or CAPD could not be randomized, and therefore our data are potentially biased by multiple factors. In the analysis of the baseline characteristics of our two cohorts (CAPD and HD), we carefully looked at potential confounders of NT-proBNP levels such as age, LVEF, myocardial mass. No significant difference was found in these variables in our two patient cohorts. Even though our study included all eligible patients during the given time period, it still represents a small number of individuals, and future larger studies will be important to confirm our findings. Lastly, our study could not assess whether CAPD would be a feasible alternative for large number of end stage renal disease patients, as this dialysis modality is dependent on the abiliteis of each patient as well as on the regional availability of such treatments.

\section{CONCLUSION}

Significantly more patients treated by CAPD have normal values of circulating levels of NT-proBNP compared to those treated by haemodialysis. This difference is most pronounced in patients with normal left ventricle ejection fraction. These findings suggest that large volume shifts during HD lead to chronically elevated levels of NT-proBNP, which in turn may predict adverse cardiovascular prognosis. Future studies are necessary to identify the most appropriate patients in whom CAPD should be preferred to HD.

\section{ABBREVIATIONS}

ACEI, Angiotensine converting enzyme inhibitors; ARB, Angiotensine receptor blockers; ASA, Acetylsalicylic acid; BB, Betablockers; BNP, B-type of natriuretic peptide; CAPD, Continuous ambulatory peritoneal dialysis; CCB, Calcium channel blockers; CTR, Cardiothoracic ratio; DBP, Diastolic blood pressure; ELISA, Enzyme-linked immunosorbent assay; HD, Hemodialysis; HR, Heart rate; IVSDd, Interventricular septum diastolic diameter; LAD, Left atrium diameter; LVDd, Left ventricle diastolic diameter; LVDs, Left ventricle systolic diameter; LVEF, Left ventricle ejection fraction; LVM, Left ventrcile mass; MI, Myocardial infarction; NT-proBNP, N-terminal fragment of pro brain natriuretic peptide; NYHA class, New York Heart Association classification; PWDd, Posterior wall diastolic diameter; $\mathrm{R}_{\mathrm{s}}$, Spearman 's correlation coefficient; SBP, Systolic blood pressure.

\section{CONFLICT OF INTEREST STATEMENT}

Author's conflict of interest disclosure: The authors stated that there are no conflicts of interest regarding the publication of this article.

\section{REFERENCES}

1. Maisel A. The coming of age of natriuretic peptides: the emperor does have clothes! J Am Coll Cardiol. 2006;47:61-4.

2. Hammerer-Lercher A, Puschendorf B, Mair J. Cardiac natriuretic peptides: new laboratory parameters in heart failure patients. Clin Lab 2001;47:265-77.

3. Gegenhuber A, Mueller T, Firlinger F, Lenz K, Poelz W, Haltmayer $M$. Time course of B-type natriuretic peptide (BNP) and N-terminal proBNP changes in patients with decompensated heart failure. Clin Chem 2004;50:454-6.

4. Yamamoto K, Burnett JC Jr, Bermudez EA, Jougasaki M, Bailey KR, Redfield MM. Clinical criteria and biochemical markers for the detection of systolic dysfunction. J Card Fail 2000;6:194-200.

5. Maisel AS, Koon J, Krishnaswamy P, Kazenegra R, Clopton P, Gardetto N, Morrisey R, Garcia A, Chiu A, De MA. Utility of B-natriuretic peptide as a rapid, point-of-care test for screening patients undergoing echocardiography to determine left ventricular dysfunction. Am Heart J 2001;141:367-74.

6. Vasan RS, Benjamin EJ, Larson MG, Leip EP, Wang TJ, Wilson PW, Levy D. Plasma natriuretic peptides for community screening for left ventricular hypertrophy and systolic dysfunction: The Framingham heart study. JAMA 2002;288:1252-9.

7. Tateishi J, Masutani M, Ohyanagi M, Iwasaki T. Transient increase in plasma brain (B-type) natriuretic peptide after percutaneous transluminal coronary angioplasty. Clin Cardiol 2000;23:776-80.

8. Kikuta K, Yasue H, Yoshimura M. Increased plasma levels of B-type natriuretic peptide in patients with unstable angina. Am Heart J 1996;132:101-7.

9. Goetze JP, Christoffersen C, Perko M, Arendrup H, Rehfeld JF, Kastrup $J$, Nielsen LB. Increased cardiac BNP expression associated with myocardial ischemia. FASEB J 2003;17:1105-7.

10. Gardner RS, Ozalp F, Murday AJ, Robb SD, McDonagh TA. N-terminal pro-brain Natriuretic Peptide a New Gold Standard in Predicting 
Mortality in Patiens with Advanced Heart Failure. Eur Heart $J$ 2003;24:1735-43.

11. Nielsen LS, Svanegaard J, Klitgaard NA, Egeblad H. N-terminal probrain Natriuretic Peptide for discriminating between Cardiac and Non-cardiac Dyspnoea. Euro. J. Heart Failure 2004;6:63-70.

12. Tsumamoto T, Wada A, Maeda K. Attenuation of compensation of endogenous cardiac natriuretic peptide system in chronic heart failure: prognostic role of plasma brain natriuretic peptide concentration in patiens with chronic symptomatic left ventricular dysfunction. Circulation 1997:96:509-16.

13. Januzzi JL Jr, Camargo CA, Anwaruddin S. The N-terminal pro-BNP investigation of dyspnea in the emergency department (PRIDE) study. Am J Cardiol 2005;95:48-54.

14. Kazanegra R, Cheng V, Garcia A, Krishnaswamy P, Gardetto N, Clopton P, Maisel A. A rapid test for B-type natriuretic peptide correlates with falling wedge pressures in patients treated for decompensated heart failure: A pilot study. J Card Fail 2001;7:21-9.

15. Rahman M, Dixit A, Donley V, Gupta S, Hanslik T, Lacson E, Ogundipe A, Weigel K, Smith KC. Factors associated with inadequate blood pressure control in hypertensive hemodialysis patients. Am J Kidney Dis 1999:33:498-506.

16. Gunal Al, Duman S, Ozkahya M, Toz H, Asci G, Akcicek F, Basci A. Strict volume control normalizes hypertension in peritoneal dialysis patients. Am J Kidney Dis 2001;37:588-93.

17. Koc M, Toprak A, Tezcan H, Bihorac A, Akoglu E, Ozener IC. Uncontrolled hypertension due to volume overload contributes to higher left ventricular mass index in CAPD patients. Nephrol Dial Transplant 2002;17:1661-6.

18. Konings $C J$, Kooman JP, Schonck M, Dammers R, Cheriex E, Palmans Meulemans AP, Hoeks AP, van Kreel B, Gladziwa U, van der Sande FM, Leunissen KM. Fluid status, blood pressure, and cardiovascular abnormalities in patients on peritoneal dialysis. Perit Dial Int 2002;22:477-87.

19. Foley RN, Parfrey PS, Sarnak MJ. Epidemiology of cardiovascular disease in chronic renal disease. J Am Soc Nephrol 1998;9:S16-S23.

20. Ludka O, Spinar J, Vitovcova L, Sobotova D, Spinarova L, Pozdisek
Z, Musil V, Vitovec J, Tomandl J. Variability of plasmatic levels of big endothelin and NT-proBNP in patients with heart failure in a chronic haemodialysis programme. Vnitr Lek 2007;53(11):1177-81.

21. Lang CC, Choy AM, Henderson IS. Effect of haemodialysis on plasma levels of brain natriuretic peptide in patients with chronic renal failure. Clin Sci (Lond) 1992;82:127-31.

22. Horl WH. Natriuretic peptides in acute and chronic kidney disease and during renal replacement therapy. J Investig Med 2005;53:36670.

23. Štejfa M a kol. Kardiologie. Grada; 1998. P. 492.

24. Khalifeh N, Haider D, Horl WH. Natriuretic Peptides in Chronic Kidney Disease and During Renal Replacement Therapy: An Update. Journal of Investigative Medicine 2009;57(1):33-9.

25. Dhar S, Pressman GS, Subramanian S, Kaul S, Gollamudi S, Bloom EF, Figueredo VM. Natriuretic peptides and heart failure in the patient with chronic kidney disease: a review of current evidence. Postgrad Med J 2009;85:299-302.

26. Wahl HG, Graf S, Renz H, Fassbinder W. Elimination of the Cardiac Natriuretic Peptides B-Type Natriuretic Peptide (BNP) and N-Terminal proBNP by Hemodialysis. Clinical Chemistry 2004;50(6):1071-3.

27. Nakatani T, Naganuma T, Mazura C, Uchida J, Sugimura T, Sugimura K. Significance of brain natriuretic peptides in patients on continuous ambulatory peritoneal dialysis. International Journal of Molecular Medicine 2002;10:457-61.

28. Al-Hweish A, Sultan SS, Mogazi K, Elsammak MY. Plasma myeloperoxidase, NT-proBNP, and troponin-I in patients on CAPD compared with those on regular hemodialysis. Hemodial Int 2010;14(3):308-15.

29. Sanjuan R, Oliva SM, Blasco ML, Puchades M, Torregrosa I, Ramón RG, Carrasco AM. Plasma Brain Natriuretic Peptide Levels in Cardiac Function Assessment in Chronic Dialysis Patients. Cardiorenal Med 2011;1(3):147-55

30. Bavbek N, Akay H, Altay M, Uz E, Turgut F, Uyar ME, Karanfil A, Selcok $Y$, Akcay A, Duranay M. Serum BNP concentration and left ventricular mass in CAPD and automated peritoneal dialysis patients. Perit Dial Int 2007;27(6):663-8. 